\title{
A Benchmarked Evaluation of a Selected CapitalCube Interval-Scaled Market Performance Variable
}

\author{
Edward J. Lusk ${ }^{1}$ \\ ${ }^{1}$ The State University of New York (SUNY) at Plattsburgh, 101 Broad St., Plattsburgh, NY, USA \& Emeritus \\ Department of Statistics: The Wharton School: University of Pennsylvania, Phila. PA, USA \\ Correspondence: E. Lusk, SBE SUNY Plattsburgh, 101 Broad St. Plattsburgh, NY, USA 12901
}

Received: February 8, 2019

Accepted: March 1, 2019

Online Published: March 5, 2019

doi:10.5430/afr.v8n2p1

URL: https://doi.org/10.5430/afr.v8n2p1

\begin{abstract}
Context In this fifth analysis of the CapitalCube ${ }^{\mathrm{TM}}$ Market Navigation Platform[CCMNP], the focus is on the CaptialCube Closing Price Latest [CCPL] which, is an Interval Scaled Market Performance [ISMP] variable that seems, a priori, the key CCMNP information for tracking the price of stocks traded on the S\&P500. This study follows on the analysis of the CCMNP's Linguistic Category MPVs [LCMPV] where it was reported that the LCMPV were not effective in signaling impending Turning Points [TP] in stock prices. Study Focus As the TP of an individual stock is the critical point in the Panel and was used previously in the evaluation of the CCMNP, this study adopts the TP as the focal point in the evaluation montage used to determine the market navigation utility of the CCPL. This study will use the S\&P500 Panel in an OLS Time Series [TS] two-parameter linear regression context: $\mathrm{Y}[\mathrm{S} \& \mathrm{P} 500]=\mathrm{X}[$ TimeIndex $]$ as the Benchmark for the performance evaluation of the CCPL in the comparable OLS Regression: Y[S\&P500] = X[CCPL]. In this regard, the inferential context for this comparison will be the Relative Absolute Error [RAE] using the Ergodic Mean Projection [termed the Random Walk[RW]] of the matched-stock price forecasts three periods after the TP. Results Using the difference in the central tendency of the RAEs as the effect-measure, the TS: S\&P Panel did not test to be different from the CCPL-arm of the study; further neither outperformed the RW; all three had Mean and Median RAEs that were greater than 1.0 — the standard cut-point for rationalizing the use of a particular forecasting model. Additionally, an exploratory analysis used these REA datasets blocked on: (i) horizons and (ii) TPs of DownTurns \& UpTurns; this analysis identified interesting possibilities for further analyses.
\end{abstract}

Keywords: CapitalCube Price Latest, Turning Points, S\&P500

\section{Introduction}

\subsection{Context of this Research Report}

The focus of this research report is to follow-up on the research reports of Lusk \& Halperin (2015, 2016 \& 2017) which addressed the nature of the associational analysis of selected variables of the CapitalCube ${ }^{\mathrm{TM}}$ Market Navigation Platform [CCMNP: [<http://www.capitalcube.com/ $>$ ] a commercial product of AnalytixInsight ${ }^{\mathrm{TM}}$ [<http://www.analytixinsight.com/ $>$ ]. For the CCMNP variable-set selected, they report that the Nulls of their interand intra-group associations may be rejected in favor of the likelihood that these CCMNP variables are not produced by random generating processes. Simply, there is structural association in the Chi2 \& Pearson Product Moment context, referencing the usual Nulls, for the Linguistic Market Performance [LMP] variables and the Interval Scaled Market Performance [ISMP] variables tested. Following on this information, the next step in the systematic evaluation of the CCMNP was then: Given that there is evidence of non-random association for various arrangements of the LMP \& the ISMP variables, does this structure create information that would empower decision makers who are using the CCMNP to make market decisions? This lead to the next study, that of Lusk (2018), where 12 LMP-variables were tested for their impact on providing information for detecting impending Turning Points [TP] in the S\&P500. For example, one of the LMP-variables offered in the CCMNP is: Accounting Quality that has four Linguistic Qualifiers [LQ] [Aggressive Accounting, Conservative Accounting, Sandbagging, Non-Cash Earnings]. This LMP and the related LQs were among eleven others tested to determine if these twelve LMP[LQ] variables contained information useful in detecting impending S\&P500 TP - a change in the trajectory of the S\&P500 value. 
The summary of the Lusk (2018) study is that: The CCMNP does NOT provide information from its LMP[LQ] variable-set that would flag or signal an impending TP.

This, of course, leads to the next study, which is the point of departure of this study for which a question of interest is:

Do the Set of Interval Scaled Market Performance [ISMP] Variables provide forecast acuity for time periods after a detected TP?

This, then, is a corollary to the Lusk(2018) paper. Lusk (2018) found that the CCMNP set of linguistic variables was not likely to identify a TP from the currently available information of the CCMNP. This study then asks:

What-If a Decision Maker could have ferreted out from all the available information that a particular month would be a TP, is there an ISMP-variable in the CCMNP that would allow the DM to forecast the stock price a few periods after the TP that would outperformed using just a Time Series projection?

This question will form the nexus of this research. The rationale underlying this study is to determine if the variables offered in the CCMNP are sensitive to future trajectory changes in the market for selected firms. If this is not the case then it would be difficult to justify the allocation of time and resources in using the CCMNP for the selected variable tested.

\subsection{Research Protocol}

Specifically, we will:

1. Reiterate the computational definition of a Turning Point [TP] used in Lusk(2018),

2. Rationalize the selection of the CCMNP ISMP-variable that will be tested for its impact in forecasting into the near horizon after the TP,

3. Describe and defend the forecasting context and the RAE measure for judging the acuity of the selected ISMP-variable in providing useful forecasts for the periods after an impending TP,

4. Detail an inference testing protocol and the operative hypothesis for evaluating the utility of the information provided by the CCPL insofar as forecasting effectiveness is concerned,

5. Discuss the results and summarize the impact of this study, finally

6. Offer suggestions for future studies addressing the forecasting acuity of a MNP.

\section{Turning Point: The Litmus Test for a MNP}

\subsection{Measures of Predicative Acuity}

Accepting that MNPs must justify their cost, a forecasting context fits perfectly re: evaluating the possible dollar-value gain garnered from an effective forecasting model vis-a-vis the cost of the MNP. Simply if there is forecasting acuity for the variables of the MNP then it is very likely the cost of the MNP would be a wise investment. In the Lusk (2018) paper, which evaluated 12 of the LMP Variables [LMPV] of the CCMNP, the context for a TP was adapted from the work of Chen \& Chen (2016) who focused on: Bullish turning points, i.e., "enduring" upturns. Lusk (2018) offers a slightly simpler and multi-directional calibration of a TP termed: A Dramatic Change.

\subsubsection{Dramatic Change in Direction}

For descriptive simplicity, one may think of the trajectory of a stock price as being driven by two classes of stochastic components: (i) a set of generating functions, and (ii) exogenous factors both of which are inter-dependent and non-uniformly ergodic over stochastic-discrete sections of the Panel. See Brillinger (1981). As Chen \& Chen also discuss, this presents challenges in creating filters so that the price change is indicative of an enduring structural change with-in one of the ergodic Panel segments. Also see Nyberg (2013, p. 3352). In this regard, Lusk (2018) selected the following measure, SRC, which will be used in this paper. Lusk (2018) offers that the SRC is: relevant, reliable, and independent - non-conditioned on the MNP Screen profiles - and so is a reasonable measure of the change of a stock price valued at the bell-price:

$$
\text { Signed Relative Change }[\mathrm{SRC}]=\frac{\left[\frac{\sum Y_{t+i}}{n}-Y_{t}\right]}{Y_{t}}
$$

where: $Y_{t}$ is monthly average reported by WRDSTM for the S\&P500 at month, $t, \mathrm{n}=4, i=, 1,2,3,4$. 
Additionally, as does Chen \& Chen, it is necessary for a screening protocol to identify an important change in trajectory in the stock trading profile; to this end a Dramatic TP is recorded if the Abs[SRC] $>25 \%$.

\subsubsection{TP Discussion}

The screen for the SRC is a simple short-term Smoothing filter in the Mean-Class. In this case, given the expected stochastic variation in an auto-correlated environment, stocks prices in actively traded markets are the classic example of Fixed Effects variables; thus, it is expected that the longer the filter the more TPs will be created using EQ1. And by symmetry the shorter the Smoothing section the less TPs will be created. For example, for the stock CUMMINS INC, [CMI] over the S\&P500 Panel from 2005 through 2013, the SRC flags 17.3\% of the months as TPs over the rolling S\&P500 SRC-screen. If one doubles the SRC-Screen to 8 months the percentage of SRC flags goes to $27.6 \%$ a $59.5 \%$ Increase. If one reduces SRC-Screen by $50 \%$, the number of TPs flagged is $11.2 \%$ a reduction of $35.3 \%$. In the case of calibrating the SRC, one seeks a balance. As the decision-maker will need to use the TP information to effect action plans, a four month waiting period seems to be in the "Goldilocks Zone": Not too long: Not too short: Just right. Therefore, the Lusk (2018) calibration as scripted in SRC: EQ1 (Note 1) seems reasonable.

\subsection{The TP Question of Interest}

However, to be clear: The definition of a TP fixes the TP in the past relative to the current set of data. To this extent, this is NOT likely to be a practical construct in the dynamic market trading world. This is NOT a problem as a more basic question is posed:

What-If the DM were to have flagged a particular month as a TP-ignoring for the moment HOW the DM would actually effect such an identification? IF the DM were to know a month to be a TP, are there CCMNP ISMP variables that would be useful in creating an effective forecast of the likely S\&P500 value in the short run, three periods ahead? IF so, then this would rationalize the search for a model of TP detection and then with a likely TP, so flagged, the CCMNP variable of interest could be used to form a forecast assuming that it were to be more effective than a forecast from a simple Time Series forecasting model.

\section{The Interval Scaled Market Performance Set Selected From the CCMNP}

\subsection{CCMNP Possible Variables}

Four possible interval scaled decision-making variables were identified that are sufficiently populated in the CCMNP that could be used as the proto-desirable variables for the test of the CCMNP:

1. Current Price Level Annual [CPLA]; This is a ratio formed as the bell-price on a particular day as benchmarked by the Range of previous trading-day values going back one year in time. This results in the CPLA being a very long-memory smoothed variable; in other words the CPLA is an Ergodic Mean level projection scaled to the fuzzy-interval: $[\approx 0$ to $\approx 1]$. As a long-memory filter in the Moving Average class, prima fascia, it would lack the temporal sensitivity to qualify as a reasonable evaluation of TP acuity.

2. Previous Day Closing Price Latest [PDCPL]: Is the bell-price adjusted for stock splits and any sort of stock spin-offs going back a number of years. This is effectively an isomorphic associative variable to the S\&P500 assuming that the market is making the same sort of re-calibrations. This is usually the case which is the underlying rational for the Sharpe (1964) CAPM as a volatility benchmark using the OLS-regression focusing on the slope or $\beta$. This high association is exactly what Lusk \& Halperin (2015) find and report in the association of the PDCPL with the value for the stock on the S\&P500. The PDCPL, as reported by Lusk (2018), with rare exceptions had Pearson Product Moment associations with the S\&P500 that were > V.5 - the Harman (1960) factor cut-off for meaningful rotation. Therefore, there is no productive information in the PDCPL vis-à-vis the S\&P500 relative to impending TPs.

3. Scaled Earnings Score Average Latest [SESAL]: This starts with the reported earnings of the firm and uses a number of context variables such as Working Capital; Earnings Growth \& Revenue Growth to create an aggregate rolling benchmark that scales the reported earnings usually in the Range $[\approx 1$ to $\approx 100]$. The SESAL is more sensitive to recent activity than is the CPLA however as it is focused on a benchmark that appears to be smoothing or rolling in nature this is more of a blend of a two parameter Linear OLS: regression and an ARMIA $(0,2,2) /$ Holt model. This apparent blending uses the same logic as the aggregation model employed by Collopy \& Armstrong (1992) following on the Makridakis et als. (1982) study. So this is a possible variable as it does offer relative end-point sensitivity compared to the CPLA. However, the SESAL has a revenue component bias. Thus the SESAL may be too focused on revenue impact effects on S\&P500. If it were to be the case that Revenue was the dominate driver of the S\&P then 
this variable would have been a viable and desirable candidate. However, there is little research support for a revenue partition given the extended Market Cap results work of Fama and French (1992, 2012).

4. CapitalCube Price Latest [CCPL]: This is a projective rolling variable-i.e., longitudinal adjusted-for Split/Spins, and benchmarked by a large number of market performance measures. The CCPL is projective in nature and used, for example, to index the Under-and Over-Priced labeling of the CCMNP. The CCPL index-labeling employs a sensitivity analysis using a range around the mid-point of measured values of CCPL extending out to Min and Max boundaries. CCPL is a key variable that is the valuation given by the CCMNP heuristics to the stock activity. As a summary indication or an approximant projective "spot" price this seems to be the most appropriate content variable for the S\&P500 in the neighborhood of the TP. This neighborhood context, seems to be important as it is a context around, in an interval sense, the current value of the market. For this reason the CCPL, as an indicator variable, seems reasonable in a forecasting context and thus an ideal instrumental variable of the S\&P500.

\subsection{Forecasting Context for Testing Acuity}

It is not a trivial exercise to find a reasonable way to use the ISMP:CCPL variable in testing for its forecast acuity. Recall that from Lusk (2018), the LMP[LQ] did not seem to be sensitive or specific. This logically would rule out using the LMP[LQ] as a conditioning category variable for the CCPL. In this case then the model of choice not in the Mixed Multivariate modeling case, effectively the Box-Jenkins Transfer ARIMA class of models. Rather the forecasting frame seems to suggest the simple model Y[S\&P500]:X[CCPL] that could be benchmarked by the comparable Time Series model. Simply as an illustration:

Assume that a Panel of ten (10) S\&P500 stock prices the last of which is the TP \& time-matched CCPL values. If the CCPL portends a change in the S\&P500 that will happen after the TP, it should be the case that the forecast value of $\mathrm{Y}[\mathrm{S} \& \mathrm{P} 500]: \mathrm{X}[\mathrm{CCPL}]$ projected into the sub-Panel after the TP should be in-sync with the impending change. If this is the case, then we have an ideal measure of the "in-sync-ness" of the CCPL as a sensitive or informative variable. The classic measure for creating a measure of forecast acuity is offered by Armstrong \& Collopy (1992) and confirmed by recent forecasting studies such as Adya and Lusk (2016); it is called the Relative Absolute Error [RAE] of the Forecast. For example, for the assumed Panel of ten (10) of the S\&P500 values, the last of which is time indexed as: $Y_{t=10} \mathrm{t}=10$ and is also the Turning Point as well as the $\mathrm{RW}$, a forecasting model $\mathrm{f}($ ), and a one-period-ahead forecast of the S\&P500, noted as $\hat{Y}_{t+1}$. The RAE, in this case, is:

$$
R A E\left[\widehat{Y}_{t+1}\right]=\frac{\left[A B S\left[\hat{Y}_{t+1}-A_{t+1}\right]\right]}{\left[A B S\left[Y_{t=10}-A_{t+1}\right]\right]}
$$

Where: ABS is the absolute value operator, $A_{t+1}$ is the designation of the Actual value in the S\&P500 Panel at time $t+1 ; Y_{t=10}$ is the Turning Point-i.e., the S\&P500 Panel value at $t=10$ which is also the RW value.

The logic of using the RAE as a measure of forecasting acuity is intuitive. It simple says that IF the RAE is $=1.0$, the forecast error of using the TP as the one-period-ahead forecast-i.e., the RW value, gives the same forecasting error as does the forecasting model. If the RAE is $>1.0$, it indicates that the TP:RW as the forecast outperforms the forecasting model. Finally, if the RAE is $<0$, the forecasting model is better than using the TP: RW as the forecast. In the first two cases, one would reject the forecasting model and just use the "Occam's Razor" model: the TP.

\subsection{Forecasting Test Protocol}

As simple as the RAE is as a logical benchmark for forecasting acuity the creation of an inference protocol for the evaluation of the CCMNP as viewed through the CCPL requires a number of conceptual linkages to arrive at a reasonable inference structure. This montage is detailed following. There are two forecasting models that will be used to create the inferential test information. The first is the benchmarking model.

\subsubsection{Time Series Benchmark Model}

A Panel of the S\&P500 of thirteen (13) reported S\&P500 bell-prices averaged over the month as reported by CRSPTM: WRDS, the Wharton School, of the University of Pennsylvania. The first ten (10) points in the Panel are used to form the TS-forecast, the $10^{\text {th }}$ point in the S\&P500 Panel will be a TP found using the SRC formula [EQ1] recall this is also the RW value, and the last three points of the S\&P500 Panel are the holdback values that will be use to evaluate the quality of the forecasts produce by the first ten points using the RAE measure. The Variable Constructs for the TS-arm are: 
The standard two-parameter linear OLS-TS model noted as: $\mathrm{Y}\left[\mathrm{S} \& \mathrm{P}\left\{Y_{t=1},---, Y_{t=10} \equiv \mathrm{TP} \equiv \mathrm{RW}\right\}\right]=\mathrm{X}[\mathrm{Time}$ Index: $\left.\left\{X_{t=1}=1,--, X_{t=10}=10\right\}\right]$.

Projections: $\left\{Y_{t=11} ; Y_{t=12} ; Y_{t=13}\right\}$

RAE Variables: $\left\{Y_{t=11} ; Y_{t=12} ; Y_{t=13} ; A_{t=11} ; A_{t=12} ; A_{t=13} ; Y_{t=10} \equiv \mathrm{TP} \equiv R W\right\}$

This TS-context provides a benchmark for the test of the CCPL. This TS-benchmark is a forecast projection after the TP for three (3) one-period ahead forecasts using only the neutral instrumental variable: the Time Index. The RAE computation is then the answer to the question: What is the RAE-forecasting error of the TS-forecasting model projections? This is a perfect benchmark as the same RAE computation will be made for the Y:X OLS-regression forecasting model using the CCPL; thus the relationship of the models [TS \& Y:X] relative to their RAE-test values will be a direct test of the effect of the acuity of the CCPL; also of interest: this design permits the use of a Matched inferential model which is more powerful. Consider now the Y[S\&P500] $=\mathrm{X}[\mathrm{CCPL}]$ version.

\subsubsection{Y $[S \& P 500]=X[C C P L]$ Model}

As for the CCPL-arm of the study, that will be benchmarked by the TS-model the same OLS model will be used for a two-stage projections: (i) the Projection of the CCPL three periods-ahead and (ii) the Y = X[CCPL] forecasts using the CCPL projections from the first stage. The two-stage projections then will be modeled as:

Stage I

Model Fit: $Y[C C P L]^{C}=\mathrm{X}[$ Time Index: $\{1,2,3,--, 10\}]$.

Projections: $\left\{Y_{t=11}^{C} ; Y_{t=12}^{C} ; Y_{t=13}^{C}\right\}$

Stage II

Model Fit: Y[S\&P500 $\left\{Y_{t=1},---, Y_{t=10} \equiv \mathrm{TP}\right\}=[\mathrm{CCPL}:\{1,2,3,--, 10\}]$

Projections: $\left\{Y_{: Y_{t=11}^{C}}^{11} ; Y_{: Y_{t=12}^{C}}^{12} ; Y_{: Y_{t=13}^{C}}^{13}\right\}$ using the projections from: $\left\{\right.$ Stage I: Projections: $\left.\left\{Y_{t=11}^{C} ; Y_{t=12}^{C} ; Y_{t=13}^{C}\right\}\right\}$.

RAE Variables: $\left\{Y_{: Y_{t=11}^{C}}^{11} ; Y_{: Y_{t=12}^{C}}^{12} ; Y_{: Y_{t=13}^{C}}^{13} ; A_{t=11} ; A_{t=12} ; A_{t=13} ; Y_{t=10} \equiv \mathrm{TP} \equiv \mathrm{RW}\right\}$.

\subsection{An Illustration}

An illustration will aid in elucidating these modeling protocols. We take up the case of: CUMMINS INC. [CMI:CCMNP designation]. The S\&P500 Panel where, using EQ1, a TP was located from the following dataset:

Table 1. CMI: S\&P500 Panel

\begin{tabular}{lllllll}
\hline 110.21 & 122.25 & 117 & 114.82 & 119.23 & 126.98 & 119.92 \\
\hline 134.56 & 134.6 & $\mathbf{1 4 4 . 7 2}$ & 92.16 & 94.23 & 101.21 & 118.70 \\
\hline
\end{tabular}

For this Panel, the Value of the SRC[EQ1[144.72]] $=-0.2981$. This was the highest value in magnitude in this Panel segment. The TP was thus flagged as: 144.72 and is bolded (Note 2). The Three Actual S\&P Holdback values are $\{92.16 ; 94.23 ; 101.21\}$ shaded in Table 1 . The Matched CCPL Panel $n=13$ was:

Table 2. CMI: CCPL Panel

\begin{tabular}{lllllll}
\hline 66.2694 & 63.21489 & 53.15605 & 55.6082 & 60.63303 & 54.22442 & 57.32492 \\
\hline 52.59557 & 50.47699 & 34.04416 & 28.42621 & 31.87145 & 47.92502 & \\
\hline
\end{tabular}

The Time Series Profile of the S\&P500[CMI] is presented in the following Table 3:

Table 3. The Information used in the TS-Profiling of the CMI.

\begin{tabular}{lll}
\hline CMI Profile & RW & TS:OLS \\
\hline Horizon1,t=11 & 144.72 & 141.3587 \\
Horizon2,t=12 & 144.72 & 144.4368 \\
Horizon3,t=13 & 144.72 & 147.5149
\end{tabular}

For Example, for Horizon2 for the OLS-TS model is: Y[Horizon2] $=144.4368=[107.4993+3.0781 \times(10+2)]$. This is produced by regressing the first $10 \mathrm{~S} \& \mathrm{P} 500$ data points in Table 1 against the matched time index $\{1,2,3$, - $-, 10\}$. 
The RAE for the info-set in Table 4 is:

Table 4. The Computation of the RAE for CMI

\begin{tabular}{llll}
\hline $\begin{array}{l}\text { CMI Profile } \\
\text { TS-Model }\end{array}$ & $\begin{array}{l}\text { RAE: } \\
\text { Numerator }\end{array}$ & $\begin{array}{l}\text { RAE: } \\
\text { Denominator }\end{array}$ & $\begin{array}{l}\text { RAE: Value } \\
\text { OLSR[CMI] }\end{array}$ \\
\hline Horizon1 & 49.1987 & 52.5600 & 0.9361 \\
Horizon2 & 50.2068 & 50.4900 & 0.9944 \\
Horizon3 & 46.3049 & 43.5100 & 1.0642 \\
Average & N/A & N/A & 0.9982 \\
\hline
\end{tabular}

Computational Illustration For Horizon2 is:

RAE: Numerator: ABS[144.4368 - 94.23] = 50.2068

RAE: Denominator: ABS[144.72 - 94.23] = 50.4900

RAE Value: ABS[144.4368 - 94.23] / ABS[144.72 - 94.23] = 50.2068 / 50.4900 = 0.9944

The average RAE for CMI regarding the Time Series projection using only the S\&P500 and the neutral X-variable of the Time index is certainly in the inference neighborhood of 1.0 indicating that for CMI one could just use the RW or the last observed value of the S\&P500 of 144.72 in the stead of the Time Series: Y $=[S \& P 500:\{1,2,3,---$, 10 \}forecasting model.

This Time Series will be used as the benchmark the following Two-stage regression.

\subsubsection{The CCPL-arm of the Computation for the Two-Stage Model}

The same dataset for CMI as presented in Tables $1 \& 2$ is used. In this case, the first set of computations are to produce the Stage I information for the CCPL. In this case, the fitted Time Series model for the CCPL values in Table 5 form the following three period ahead projections:

Table 5. CMI Projected Values

\begin{tabular}{ll}
\hline Projected CCPL Values & CCPL \\
\hline Horizon1 & 41.9797 \\
Horizon2 & 39.6569 \\
Horizon3 & 37.3342
\end{tabular}

For example, the projected value of the CCPL for the second projected period ahead is: the Y $[$ Horzon2] $=\mathbf{3 9 . 6 5 6 9}=$ [67.52989- $2.3228 \times(10+2)]$.

With this information one can now make the projection of the S\&P500 values given the CCPL values as input to the Second stage modeling phase. The value of the S\&P500 projections using the CCPL values in Table 6 are:

Table 6. S\&P500 Projections

\begin{tabular}{ll}
\hline Projected S\&P500[CCPL] Values & S\&P500 \\
\hline Horizon1 & 137.3287 \\
Horizon2 & 139.6741 \\
Horizon3 & 142.0195
\end{tabular}

For example, the value for Horizon2, is found by using the CCPL projection of 39.6569 in the regression of $\mathrm{Y}[\mathrm{S} \& \mathrm{P} 500]=\mathrm{f}[\mathrm{CCPL}]$ for the first ten Panel values from Table 1 for the S\&P values and Table 2 for the CCPL values. This regression has an Intercept of: 179.7178 and Slope of -1.0098 . This produces the values in Table 6. For example, for Horizon2:

\section{Horizon2 for the $\mathbf{S \& P}$ is $139.6741=[179.7178-1.0098 \times(\mathbf{3 9 . 6 5 6 9})]$}

This now gives the information that is needed to compute the Y:X RAE for the S\&P500 projections. These RAE are noted following: 
Table 7. CMI S\&P500 RAE for the Projections

3.4.2 Computational Illustration

\begin{tabular}{ll}
\hline CMI S\&P500 RAE & RAE \\
\hline Horizon1 & 0.8594 \\
Horizon2 & $\mathbf{0 . 9 0 0 1}$ \\
Horizon3 & 0.9379 \\
\hline
\end{tabular}

For Horizon2 is:

RAE: Numerator: ABS[139.6741-94.23] = 45.4441

RAE: Denominator: ABS[144.72 - 94.23] = 50.4900

RAE Value: ABS[139.6741 - 94.23] / ABS[144.72 - 94.23] = [45.4441/ 50.4900 $]=\mathbf{0 . 9 0 0 1}$

3.5 Pedagogical Note:

The only change in moving from the Time Series to the $\mathrm{Y}: \mathrm{X}$ regressions is the change in the numerator of the RAE computation as the only change in the projection of the forecasted value. In the Time Series modeling context, the Horizon2 projection was: 144.4368 while for the Y:X[CPPL] projection this was: 139.6741 . As the latter value is closer to the actual value of 94.23 , the RAE is lower for the $\mathrm{Y}: \mathrm{X}$ regression.

Now there are two sets of RAEs for the CMI trial, one of the Time Series projection for the S\&P500 and the other for the S\&P500[CMI] projections using the CCPL projected values. These are presented in Table 8:

Table 8. Benchmark and CCPL RAE for S\&P500[CMI]

\begin{tabular}{lll}
\hline S\&P500[CMI] & RAE S\&P500 TimeSeries & RAE Y:X S\&P500 CCPL \\
\hline Horizon1 & 0.9361 & 0.8594 \\
Horizon2 & 0.9944 & 0.9001 \\
Horizon3 & 1.0642 & 0.9379 \\
Average & 0.9982 & 0.8991
\end{tabular}

These sets of RAE will be used to address the question: If one were to just use the S\&P500 values for the TS-model and then using these same datasets, forecasts are produced for the two-staged Y:X model using the CCPL, do the RAEs of the two-staged model outperform those just using the S\&P500 as a Time Series projection?.

Using the information in Table 8, one observes that the CCPL seems to add predictive acuity. Interestingly, but as expected, the RAE are increasing over the projection time frame. This is a vetting check in that it is almost axiomatic that forecast accuracy declines [forecast error increases] as the projection period increases. This is the profile for the CMI dataset. Also the RAE for the S\&P500 is, for this example, always greater than the RAE using the CCPL to condition the projection. This means that the S\&P500 values projected using the CCPL projection were uniformly closer in absolute measure to the S\&P500 Holdback values: $\left\{A_{t=11}=92.16 ; A_{t=12}=94.23 ; A_{t=13}=\right.$ 101.21\}than were the S\&P500 measures in the TS-context i.e., where there was NO other conditioning information. This is presented in the following Table 9 as an illustrative clarification of the RAE measures.

Table 9. Summary of the CMI TS-Benchmark \& the Y:X[CCPL] Regression

\begin{tabular}{lllllll}
\hline & RW=TP & $\begin{array}{l}\text { S\&P500 } \\
\text { TimeSeries }\end{array}$ & $\begin{array}{l}\text { S\&P500 } \\
\text { CCPL }\end{array}$ & $\begin{array}{l}\text { S\&P500 } \\
\text { Actual }\end{array}$ & $\begin{array}{l}\text { Forecast } \\
\text { Error TS }\end{array}$ & $\begin{array}{l}\text { Forecast } \\
\text { Error CCPL }\end{array}$ \\
\hline Horizon1 & 144.72 & 141.3587 & 137.3287 & 92.16 & 49.1987 & 45.1687 \\
Horizon2 & 144.72 & 144.4368 & 139.6741 & 94.23 & 50.2068 & 45.4441 \\
Horizon3 & 144.72 & 147.5149 & 142.0195 & 101.21 & 46.3049 & 40.8095 \\
Average & 144.72 & 144.4368 & 139.6741 & 95.8667 & 48.5701 & 43.8074
\end{tabular}

Therefore, as the Time Series projection is uniformly further away from the S\&P500 actual values and recognizing that the numerator of the RAE is impacted by this magnitude then it is easy to see whey the RAE is larger for the S\&P500 unaided by the CCPL information. The CMI analysis was offered to illustrate the RAE computations and 
demonstrate the logic of the RAE as a generalizable benchmark of forecast acuity. With this as context consider now the inferential analysis of the CCPL as a CCMNP variable of interest to decision makers.

\section{Hypotheses and Testing Protocols}

\subsection{Principal Test}

The first inferential test establishes the base line for the central question of this research: $H$, here stated in the Alternative Form-i.e., assuming that the CCPL is an informed and useful variable:

Ha In the context of an assumed [detected] Turning Point[TP], as defined above, using the RAE of the forecast for inference testing, using the CCPL as a conditioning variable for the OLSR-Y:X projection into the short-term Panel Space after the TP, and finally benchmarking the Y:X result with the OLSR-TS result, it is expect that: The central tendency of the respective RAE[Y:X] and the RAE[TS] will be different.

\subsubsection{Testing Rationale Discussion}

Initially, one needs to specify the inference testing protocol for the Null of $\mathrm{Ha}$. One innovative approach for judging performance differences in experimental situations is to examine the probability of the False Negative Error [FNE] - i.e., What is the jeopardy of "believing" that the Null of No Difference is the likely State of Nature when, in fact, that may not be the case? What makes the FNE a reasonable evaluation inferential measure is: (i) the FNE is fixed in the actual experimental situation relative to an "Alert or Alternative" belief which is the usual test-against value, and (ii) the FNE risk is high[low] when the difference between the hypothesized value and the test-against Alert-value is small[large]. This is "natural feedback" on the futility of trying to nuance the detection parameters with an unrealistic degree of precision for a particular experimental design as this invites a High FNE where the DM will usually fall into the Non-Rejection region, which is the Left-Hand-Side [LHS] between $-\infty$ and the FPE probability cut-point relative to the distribution centered at $\mu_{\alpha}$ assuming that mean shifts do not change the variance structure. To compute the FNE requires specification of: (i) the False Positive Error[FPE] testing protocol, (ii), the historical/reasonable mean value: $\mu_{o}$, (iii) the Alert test-against value noted as: $\mu_{\alpha}$, and (iv) of course: collectively the sample size, standard deviation of the sample, and the assumption of the data generating process. In this case, then one can compute the FNE. For example, assuming: a Normal generating process, a typical RAE-test of $\mu_{o}=1.0$, a directional FPE of $\alpha \%=5 \%$, and that the analyst proffers a reasonable $\mu_{\alpha}$ would be $\mu_{\alpha}=1.25$ : e.g., $\left[\mu_{o}+\right.$ $\mu_{o} \times 25 \%$ ], then one could compute the FNE for this particular forecasting model. Specifically, for a test of Horizon1 for the RAE[Y:X] configuration, the actual information set was: $s_{Y: X}=0.5792$ and $\mathrm{n}=32$; this gives a $s_{\varepsilon}$ of: $0.1024[0.5792 / \sqrt{32}]$. If it is proposed that $\mu_{\alpha}=1.25$ and for a directional FPE greater-than $p$-value cut-point of 5\%, the FNE of the experimental configuration using the test-against value is defined as:

$$
P\left[t<t_{\alpha, d f}-\left[\frac{A B S\left[\mu_{o}-\mu_{a}\right]}{s_{\varepsilon}}\right]\right]
$$

Or, in this case the overlap of the Non-Rejection FPE region [LHS] will be:

$$
\begin{gathered}
P\left[t<\operatorname{Excel}[\text { T. INV }(95 \%, 31)]-\left[\frac{A B S[1.0-1.25]}{0.1024}\right]\right] \\
P[t<1.6955-2.4417] \\
P[t<-0.7461]=[1-\operatorname{Excel}[\text { T.DIST.RT }(-0.7461,31)]=\mathbf{2 3 . 0 6 \%}
\end{gathered}
$$

This then is the FNE (Note 3) of the LHS-overlap where the analyst fails to detect that $\mu_{o}$ is not likely the case in favor that the population mean is $>\mu_{o}$.

While the FNE seems to be a useful, and to be sure innovative, inferential measure in this forecasting context there is a possible judgmental bias. Recall that there are two arms: The TS and the $\mathrm{Y}: \mathrm{X}$. In this case, then an alternative Test Against value would logically have to be developed for each arm considering their design effects; in this case $\mu_{a}=$ 1.25 is reasonable choice for both arms. Then one would need to have a Test Against measure for the comparison of the FNEs between the arms as well. This is where there could be a judgmental bias as there is no precedent studies to use for guidance for the comparison of the two FNE-profiles both of which would have to be iterated over many sample sets or bootstrapped. To avoid these issues, a more direct analysis has been selected. As the two arms of the study use the same S\&P500 datasets and each is benchmarked using the same RAE-protocol, a Matched RAE design is possible. This has the advantage of increasing Power and so the differential result will be more precise. Note also that $H a$ offers a non-directional test. This was selected as: (i) there is nothing restricting the RAE results from either of the two directional effects, (ii) either of the directional effects would be valuable market navigation information, 
and (iii) a one directional test often creates an illusion of precision as the rejection of the Null occurs more readily as the $\alpha$-cut-point is lower.

\subsubsection{Testing Protocol}

The simplest test of $\mathrm{Ha}$ referencing the Null as the test concept for each of the blocked horizons is a standard Paired/Matched t-test. This has the best Power relative to the Random Effects profile and the model blocking suits the matching assumptions and so is a natural and desirable variance control. As a point of information, for the general context using the SAS:JMPv.13 DOE Power Platform, the Power [using the average of the standard deviation over the three horizons, a non-directional test of a 5\% effect-value, and 0.25 detection span] is $94.992 \%$ which gives_a FNE of approximately 5\% or in this case an almost "perfectly" balanced FPE\&FNE design.

\subsection{Results}

Sixteen (16) firms [see the Appendix] were randomly sampled from the CCMNP for which there were, in total, 32 TPs identified using the Dramatic TP protocol. Using the testing protocols discussed above, the following test information was generated for: Y[S\&P500] $=[C C P L:\{1,2,3,--, 10\}] \&$ Y $[S \& P 500]=[T i m e I n d e x\{1,2,3,---, 10\}]$ is presented in Table 10:

Table 10. The Results of the Testing of the CCMNP and the Benchmark using the RAE Results

\begin{tabular}{llllll}
\hline Model Tests & $\begin{array}{l}\text { Y:X } \\
\text { Mean/Median }\end{array}$ & $\begin{array}{l}\text { TS RAE } \\
\text { Mean/Median }\end{array}$ & $\begin{array}{l}\text { Inference } \\
\text { Null }\end{array}$ & {$[H a]:$} & Result \\
\hline Horizon1[Hor1] & $1.09 / 1.04$ & $1.09 / 1.07$ & Null:Not Rejected & $H a$ Not Founded \\
Horizon2[Hor2] & $1.16 / 1.06$ & $1.13 / 1.09$ & Null:Not Rejected & $H a$ Not Founded \\
Horizon3[Hor3] & $3.19 / 1.04$ & $2.98 / 1.10$ & Null:Not Rejected & $\boldsymbol{H a}$ Not Founded \\
\hline
\end{tabular}

The inference of these results is simple. The non-directional FPE: p-values using the standard Matched-Analysis from the SAS:JMPv.13 Analysis platform individually for the Horizons for [RAE[Y:X] v. RAE[TS]] are respectively: [Hor 1:95.6\%; Hor 2:70.8\%; Hor3:37.1\%]. Inference: there is no evidence that the CCPL, as a conditioning variable for $S \& P 500$, produces a population profile of RAEs with a central tendency different from that of the TS model. The Null of Ha is not rejected.

As a related analysis, another question of interest is: For the two arms, is a RAE of 1.0 in the 95\%CI for the individual horizons. If this were to be the case, there would be evidence that the forecast acuity of the Random Walk [RW] as a forecast models is no different from either: Y[S\&P500]:X[CCPL] or Y[S\&P500]:X[TimeIndex]. This was tested over the six partitions [2-Models for 3Horizons]. In this case, testing these six partitions, it is found that: All of the six partitions of the RAE results produced $95 \%$ confidence intervals that contained 1.0. This suggests that using either: Y[S\&P500]:X[CCPL] or Y[S\&P500]:X[TimeIndex] models does not outperform forecasting just using the RW S\&P500 value. Simply, the last observed value in the Panel, which is a TP, gives the same level of forecasting acuity than do the formal forecasting models.

For this matched comparison as well as the individual portions, the inference is clear: From an intuitive-i.e., all the Means and Medians are $>1.0$ and statistical perspectives,

Relative to Ha: there is no evidence that the instrumental variable CCPL has a conditioning effect on the $S \& P$ that would lead one to believe that this variable can inform the S\&P500 market trading decision compared to that of the unconditioned TS results,

Relative to the 95\%CIs Enhanced Testing, neither of the forecasting models outperforms the RW value.

This results is consistent with the research report of Lusk (2018) where the LMP variables were tested and were found not to provide information on impending TP.

\subsection{Additional Test Information}

This information is added as a descriptive elaboration. The p-values reported are situationally descriptive and are not linked to $\mathrm{Ha}$ or any design a priori context - i.e., the standard Null of no difference. It is of interest to examine, in an exploratory mode, if there are RAE differences that are related: to impending Up-Turns [UT] where: the SRC is Positive] or to impending Down-Turns[DT] [where the SRC is Negative]. Further it would be interesting to examine this question blocking on the regression models. This information is presented in Table 11 following: 
Table 11. Down-Turns v. Up-Turns for the Y:X v. TS over Horizons

\begin{tabular}{lll}
\hline Trajectory Effect & RAE[DT]:YX:TS & RAE[UT]:YX:TS \\
\hline Horizon1:Mean & $\mathbf{0 . 9 5 : 1 . 2 2 [ 0 . 0 0 0 1 ]}$ & $1.30: 0.91[0.008]$ \\
Horizon2:Mean & $1.15: 1.38[<0.0001]$ & $1.14: 0.81[0.0013]$ \\
Horizon3:Mean & $1.21: 1.45[<0.0001]$ & $5.72: 4.93[0.1409]$ \\
\hline
\end{tabular}

\subsubsection{Supplementary Results}

The codex for Table 11 is best illustrated with an example. For the shaded cell, the analysis is for the first forecasting horizon where the RAE for the Y:X regression had a mean of 0.95 and for the TS regression the mean was 1.22. The two-tailed p-value for this matched analysis was 0.0001 indicating that the Null of no difference is high unlikely to have produced this observed difference by sampling chance. These parametric p-values are basically isomorphic with Distribution Free/Non-parametric tests: the Sign-Test and Wilcoxson Signed Rank test.

\subsubsection{Discussion}

As this is an "extended exploratory analysis" it is curious that here and there are instances where the Means \& Medians are in the favorable RAE zones-i.e., <1.0. Specifically, for the bolded results for Horizon 1: for the DT the Means \& Medians are: [0.95 \& 0.92]. For the UT the Means \& Medians are: [0.91 \& 0.77]. Finally, for Horizon 2 for the UT the Means \& Medians are:[0.81 \& 0.70]. These may offer the possibility of further examination.

\section{Summary and Outlook}

\subsection{Summary}

For control purposes, the same TP-protocols found in Lusk (2018) were employed in this study. Using the CCMNP ISMP-variable: CCPL, extensive testing failed to reject the usual Nulls. Simply, there is no inferential information that points to a likely instance where the CCPL will condition the S\&P500 to produce forecasts after the TPs that are better in RAE than just using the unconditioned TS-model. This finding mirrors the results of the Lusk (2018) study where the LMP[LQ]-variables did not facilitate the detection of TPs. In addition, there was no indication that the forecasting models employed outperformed the simple RW forecast; nor is there any indication that the nature of the directional difference created by the SRC is related to the RAE profile. There are far fewer caveats for the current study than were offered in the Lusk (2018) study.

\subsubsection{Caveat Context:}

Initially, to be very clear, the testing focused on an "impossible" case in the practical market trading world. TPs are only identified in the past: ONLY after four months-A Quarter and one month-has passed. That is to say the TP is five months in the past. Then only after four months has passed is a TP flagged using the SRC[EQ1] and the $\mathrm{ABS}[25 \%]$ calibration. In the market trading world, this is probably too long to "ruminate" to take an action. To be clear, this study in not concerned with this temporal "anomaly" but depends on the following What-If question:

\subsubsection{Question of Interest}

What-If the DM found, using the historical record and some modeling protocol, that a month would eventually be a SRC-TP. In that case, this study was formed to determine if the CCPL would be useful in providing an effective forecast in the short-term after the TP compared to the simple TS-model. If this were to be the case, then this would rationalize the development of a modeling protocol to propose likely TPs so as to use the model: $\mathrm{Y}[\mathrm{S} \& \mathrm{P} 500]: \mathrm{X}[\mathrm{CCPL}]$ rather than the TS: Y[S\&P500]: X[TimeIndex]. However, the principal results of this study is that there is no RAE-difference between the Y:X or TS or, interestingly, the RW model.

\subsection{Outlook}

It is the case that many of the market navigation tools offered in the public domain are of questionable utility in the dynamic market trading world. See (Schadler \& Cotton (2008) and North \& Stevens (2015). This research report examined the utility of a reasonable a priori model-variable that, after testing, did not seem to "work-out". As the accrual was random and the sample size adequate for the matched design, only two possible design issues are suggested: (i) testing around the TP is a fool's errand, or (ii) the testing reported above was too sparse and should have included more ISMP-variables and also variables from the CCMNP-Linguistic group. As for the first, the TP, however defined, is the Gold Standard. The fact that it is difficult to find an effective forecasting model using the TP, while true, is hardly a reason to eschew such research investigations. As for the second, following on the research of Barber, Huang \& Odean (2016), a subsequent study may benefit from more extensively endowed protocols using the 
LMP[LQ] \& ISMP-variables blocked on a Super-Dramatic TP, perhaps using a cut-off of $50 \%$ rather than $25 \%$ or perhaps using a modified Chen \& Chen (2016) protocol, and then use this montage to form a TP-forecasting protocol using a mixed-multi-variate forecasting protocol based upon the RAE and, of course, benchmarked by the TS model. In the continuing analyses of MNPs perhaps using projections that are unconditioned with judgmental variables is not productive as it seems that past information alone is not able to anticipate TPs. One further line of research may be to examine if Time-Series models, in general, are a poor choice in the search for Turning Point sensitive models. If this is the case, then certainly Rule-Base judgmental models may be the best choice in decoding the market.

\section{Acknowledgments}

Thanks and appreciation are due to: Dr. H. Wright, Boston University: Department of Mathematics and Statistics, the participants at the SBE Research Workshop at SUNY: Plattsburgh, NY USA in particular Prof. Dr. K. Petrova: Department of Economics \& Finance, and Mr. Manuel Bern, Chief of Internal Audit: TUI International, GmbH, Hannover, Germany and to the two anonymous peer-reviewers of the Journal of Accounting and Finance Research for their careful reading, helpful detailed comments, and suggestions.

\section{References}

Armstrong, J \& Collopy, F. (1992). Error measures for generalizing about forecasting methods: Empirical comparisons. International Journal of Forecasting, 8, 69-81. http://dx.doi.10.1016/0169-2070(92)90008-W

Barber, B., Huang, X. \& Odean, T. (2016). Which factors matter to investors? Evidence from mutual fund flows. The Review of Financial Studies, 29, 2600-2642. http://dx.doi. 10.1093/rfs/hhw054

Brillinger. D. (1981). Time Series: Data Analysis and Theory. Holden Day: International Edition in Decision Processes $1^{\text {st }}$ Edition: ISBN-13: 978-0030769757.

Collopy, F \& Armstrong, S. (1992). Rule-Based Forecasting: Development and validation of an expert systems approach to combining time series extrapolatons. Managemnt Science, 38, 1194-1414.

Chen, T-L \& Chen, F-U. (2016). An intelligent pattern recognition model for supporting investment decisions in stock market. Information Sciences, 346/7, 261-274. http://dx.10.1016/j.ins.2016.01.079

Fama, E. \& French, K. (1992). The Cross-Section of expected stock returns. The Journal of Finance, 47, 427 - 449. http://dx.doi.org/10.1111/j.1540-6261.1992.tb04398

Fama, E. \& French, K. (2012). Size, value, and momentum in international stock returns. Journal of Financial Economics, 105, 457- 472 . http://dx.doi.org/10.1016/j.jfineco.2012.05.011

Harman, H. (1960). Modern Factor Analysis. University of Chicago Press. ISBN13: 978-022631652

Lusk, E. (2018). Evaluation of the predictive validity of the CapitalCube Market navigation platform. Accounting and Finance Research, 7, 39-59. https://doi.org/10.5430/afr.v7n3p39

Lusk, E. \& Halperin, M. (2015). An empirical contextual validation of the CapitalCube ${ }^{\mathrm{TM}}$ Market trading variables as reflected in a 10-year panel of the S\&P500: Vetting for inference testing. Accounting and Finance Research, 5, 15-26. http://dx.doi.org/10.5430/afr.v5n1p15

Lusk, E. \& Halperin, M. (2016). A linguistic examination of the CapitalCube ${ }^{\mathrm{TM}}$ market effect variables. Accounting and Finance Research, 5, 89-96. http://dx.doi.org/10.5430/afr.v5n2p89

Lusk, E. \& Halperin, M. (2017). An associational examination of the CapitalCube ${ }^{\mathrm{TM}}$ effect context for the MPV over the linguistic partitions: Testing sensitivity \& specificity. Accounting and Finance Research, 6, 1-11. https://doi.org/10.5430/afr.v6n2p1

Makridakis, S., Andersen, A., Carbone, R., Fildes, R., Hibon, M., Lewandowski, R., Newton, J., E. Parzen, E., \& Winkler. R. (1982). The accuracy of extrapolation (Time Series) methods: Results of a forecasting competition. Journal of Forecasting, 1, 111-153. http://dx.doi.org/10.1002/for.3980010202

North, D. \& Stevens, J. (2015). Investment performance of AAII stock screens over diverse markets. Financial Services Review, 24, 157-176.

Nyberg, H. (2013). Predicting bear and bull stock markets with dynamic binary time series models. Journal of Banking \& Finance, 37, 3351-3363. http://dx.doi.10.1016/j.jbankfin.2013.05.008

Schadler, F. \& Cotton, B. (2008). Are the AAII stock screens a useful tool for investors? Financial Services Review, 17, 185-201. 
Sharpe, F. (1964). Capital asset prices: a theory of market equilibrium under conditions of risk. Journal of Finance, 19, 425-442. http://dx.doi.org/10.2307/2977928

\section{Notes}

Note 1. In this study, the TPs were not prescreened to eliminate any of them that do not fit the Panel Correlation screen or the Mean test over the Pre \& Post TP sub-panel segments used in Lusk (2018). Lusk (2018) did this screening which was effectively a bias to rejecting the Null. However, for the forecasting context in this paper such a pre-conditioning or screening will be relaxed so as to eliminate the bias to favor the effect.

Note 2. Following are the $\mathrm{n}=10 \mathrm{SRC}$ values that are produced from EQ1:

$\begin{array}{lllllllllll}0.073632 & -0.02243 & 0.027671 & 0.090163 & 0.082068 & 0.050953 & 0.054953 & -0.13475 & -0.19703 & -0.29813 & \text { N/A }\end{array}$

The Point where -0.29813 was produced was 144.72. These values were included as they seem to have pedagogical impact. In the Audit \& Assurance course, many of these concepts are used; the students seem to garner insight in making these computations. Recommendation: Have the students code this rolling-screen in Excel.

Note 3. This FNE may also be decomposed as: Excel T.DIST $\left.\left[\left(R A E_{F P E}-\mu_{a}\right) / s_{\epsilon}\right], 31, T r u e\right)$ where: $R A E_{F P E}:\left[\mu_{o}\right.$ $\left.+t_{\alpha, d f} \times S_{\epsilon}\right]$. Specifically, T.DIST[-0.746148],31,True] $=23.06 \%$

Appendix

Table A1. The S\&P500 Tickers Selected Firms from the CCMNP

\begin{tabular}{|c|c|c|c|c|c|c|c|c|c|}
\hline CMI[3] & CSX[3] & DOV[1] & ETN[2] & FLS[3] & GD[2] & $\mathrm{GE}[2]$ & HON[1] & $\mathrm{MCO}[2]$ & NOC[1] \\
\hline $\mathrm{PBI}[1]$ & $\mathrm{PH}[3]$ & PWR[2] & $\mathrm{R}[2]$ & SNA[2] & $\mathrm{TXT}[2]$ & $\mathrm{N}=32$ & & & \\
\hline
\end{tabular}

